\title{
Native Landscape Plants for South Florida ${ }^{1}$
}

\author{
Jody Haynes, John McLaughlin and Laura Vasquez ${ }^{2}$
}

\section{Introduction}

Native plants were once thought of by many Florida gardeners, nurserymen, and landscapers as being appropriate only for informal gardens or in highly specific and often difficult garden situations, such as boggy or coastal areas (Osorio, 2001). Because of this negative (mis)perception, native plants have often received a "bad rap" in Florida. In recent years, however, the attributes of native plants have been increasingly recognized and appreciated especially in central and north Florida.

The Florida Yards \& Neighborhoods (FYN) program has been encouraging the use of Florida-friendly landscaping principles in south Florida since February 2000. FYN does not restrict its recommendations to native plants, but rather recommends putting the right plant in the right place. South Florida natives, by their very nature, are generally well adapted to the nutrient-poor, alkaline, and sand- or limestone-based soils of south Florida. They also have relatively low fertilizer requirements, few pest and disease problems, and typically do not require frequent maintenance -- such as regular watering, pruning, or spraying -- to remain healthy and maintain an acceptable aesthetic quality. It is also important to note here that not all native plants have the same requirements, and any plant put in the wrong place may either present problems or require more maintenance.

A previous publication, ENH854 (http://edis.ifas.ufl.edu/EP107), listed over 350 native and non-native plant species for south Florida. This new publication was developed as a supplement to ENH854, but it is also a good stand-alone reference for people wishing to add native plants to their private yards or public landscapes, or simply to learn more about them.

\section{Benefits of Native Plants}

While south Florida's native plants may not offer the striking floral displays of some tropical exotics, many do possess attractive foliage or colorful fruits. Often they can add a pleasing form or texture to the landscape. South Florida has a wide variety of native

1. This document is ENH 875, one of a series of the Environmental Horticulture Department, Florida Cooperative Extension Service, Institute of Food and Agricultural Sciences, University of Florida. Original publication date April 29, 2003. Visit the EDIS Web Site at http://edis.ifas.ufl.edu.

2. Jody Haynes, Florida Yards and Neighborhoods Program Extension Agent, Department of Environmental Horticulture, UF/Miami-Dade County Extension, Homestead, FL, e-mail jlh@mail.ifas.ufl.edu phone (305) 248-3311 x246.

John McLaughlin, Urban Horticulture Program Assistant, Department of Environmental Horticulture, UF/Miami-Dade County Extension, Homestead, FL, e-mail johnmcl@mail.ifas.ufl.edu phone (305) 248-3311 x228.

Laura Vasquez, Florida Yards and Neighborhoods Program Assistant, Department of Environmental Horticulture, UF/Miami-Dade County Extension, Homestead, FL, e-mail lavasquez@mail.ifas.ufl.edu phone (305) 248-3311 x245

Florida Cooperative Extension Service, Institute of Food and Agricultural Sciences, University of Florida, Gainesville, FL 32611

The Institute of Food and Agricultural Sciences (IFAS) is an Equal Employment Opportunity - Affirmative Action Employer authorized to provide research, educational information and other services only to individuals and institutions that function without regard to race, creed, color, religion, age, disability, sex, sexual orientation, marital status, national origin, political opinions or affiliations. For information on obtaining other extension publications, contact your county Cooperative Extension Service office. Florida Cooperative Extension Service / Institute of Food and Agricultural Sciences / University of Florida / Larry R. Arrington, Interim Dean 
plants that are both attractive and useful as landscape plants. The species listed herein grow well in urban landscape settings in part or all of south Florida without much fuss - assuming they are planted in the right place in the landscape. In addition to being interesting additions to urban yards and landscapes, native plants also increase the diversity of natural insect predators. Many are also attractive to other types of sought-after wildlife species (i.e., birds and butterflies).

\section{Finding and Using Native Plants in South Florida}

Although it is not difficult to find native plants in local garden centers, the range of species is usually extremely limited, particularly compared to the great number of exotic species available. With many local ordinances requiring an increasing percentage of native plants in new landscapes, it is becoming increasingly important that more native species be made available to the public. We have created a list of retail and wholesale nurseries in south Florida that sell native plants. If you are interested in this list, you can either download the pdf from the publications section of our website, http://miami-dade.ifas.ufl.edu/publications.htm (it is the last entry under "Trees and Landscape Plants" in our list of in-house publications), or call or e-mail us and we will send you a free copy (our contact information is given in footnote 2).

\section{County-Specific Soil Conditions}

Much of south Florida is similar with respect to climate and other growing conditions. Therefore, most of the plants on this list should be widely applicable throughout the region. However, once you pass from the sandy soils of Palm Beach, Broward, and northern Miami-Dade County into the alkaline rockland type soils of central and southern Miami-Dade and Monroe counties, the landscape environment changes dramatically, and this can affect the ability to grow certain species. Add to that the appearance of pockets of marl soil and the varying types of fill soils that can be found in urban areas, and the issue of soil compatibility becomes increasingly important. For example, buttonwood (Conocarpus erectus) will not grow well in marl soil, but excels in limestone soils. Conversely, butterfly weed (Asclepias tuberosa) will not grow well in the alkaline limestone of south Miami-Dade home landscapes, but performs quite well in the sandy areas in north Miami-Dade and further north. When applicable, we will include notes on soil preference for particular species.

\section{Native Plant Categories}

The 135 native plant species listed in this publication are grouped according to their functions in the landscape. Separate tables are provided for the following categories: wildflowers (Table 1); shrubs and small trees (Table 2); medium and large trees (Table 3); palms and our single native cycad (Table 4); ornamental grasses, ferns, and groundcovers (Table 5); and vines (Table 6). Each entry includes the common name, scientific name, maximum size, light preference, salt tolerance, distinguishing characteristics, and culture recommendations.

\section{Native Plant Resources}

Association of Florida Native Nurseries website: http://www.afnn.org.

Austin, D.F. Pine Rockland Plant Guide. Miami-Dade County Department of Environmental Resource Management, Miami, FL.

Bell, C.R. \& B.J. Taylor. 1982. Florida Wildflowers and Roadside Plants. Laurel Hill Press, Chapel Hill, NC.

Black, R.J. 1997. Native Florida Plants for Home Landscapes. University of Florida-IFAS Publication ENH-25, Gainesville.

Florida Native Plant Society website: http://www.fnps.org.

Florida Water Management Districts. 2001. Water Wise Florida Landscapes: Landscaping to Promote Water Conservation Using the Principles of Xeriscape $^{T M}$.

Floridata $^{\mathrm{TM}}$ website: http://www.floridata.com.

Haehle, R.G. \& J. Brookwell. 1999. Native Florida Plants. Gulf Publishing Co., Houston, TX. 
Meerow, A.W. 1991. Native Shrubs for South Florida. University of Florida-IFAS Publication EES-59, Gainesville.

Meerow, A.W. 1996. Native Trees for South Florida. University of Florida-IFAS Publication EES-57, Gainesville.

Meerow, A.W. 1999. Native Ground Covers for South Florida. University of Florida-IFAS

Publication EES-60, Gainesville.

Miami-Dade County. 2001. Dade County

Landscaping Manual. Miami-Dade County, FL.

Osorio, R. 2001. A Gardener's Guide to

Florida's Native Plants. University of Florida Press, Gainesville.

Taylor, W.K. 1998. Florida Wildflowers in their Natural Communities. University of Florida Press, Gainesville, FL.

Wunderlin, R.P. \& B.F. Hansen. 2000. Atlas of Florida Vascular Plants. Institute for Systematic Botany, University of South Florida. Internet: http://plantatlas.usf.edu. 
Table 1. Native wildflowers for south Florida, listed in alphabetical order by scientific name. (Note: * indicates plants commonly available in native nurseries in south Florida.)

\begin{tabular}{|c|c|c|c|c|}
\hline $\begin{array}{l}\text { Common name } \\
\text { Scientific name }\end{array}$ & $\begin{array}{c}\text { Size } \\
\text { (inches) }\end{array}$ & $\begin{array}{l}\text { Light } \\
\text { preference }\end{array}$ & $\begin{array}{l}\text { Salt } \\
\text { tolerance }\end{array}$ & Comments \\
\hline $\begin{array}{l}\text { Butterfly weed * } \\
\text { Asclepias tuberos }\end{array}$ & $18-36$ & $\begin{array}{l}\text { Full sun to } \\
\text { partial shade }\end{array}$ & Low & $\begin{array}{l}\text { Perennial wildflower with orange summertime flowers. } \\
\text { Essential component of a butterfly garden. Attracts } \\
\text { butterflies and hummingbirds. Prefers sandy soil; will not } \\
\text { grow well in alkaline limestone soil. }\end{array}$ \\
\hline $\begin{array}{l}\text { Silver oxeye * } \\
\text { Borrichia } \\
\text { frutescens }\end{array}$ & $2-4$ & Full sun & High & $\begin{array}{l}\text { Similar to } B \text {. arborescens, but with silvery foliage and a less } \\
\text { upright stature. }\end{array}$ \\
\hline $\begin{array}{l}\text { Twinflower * } \\
\text { Dyschoriste } \\
\text { oblongifolia }\end{array}$ & $8-12$ & $\begin{array}{l}\text { Full sun to } \\
\text { partial shade }\end{array}$ & Low & $\begin{array}{l}\text { Small, sprawling perennial that forms a groundcover by } \\
\text { underground rhizomes and seeds. Delicate stems bear } \\
\text { small purple flowers. }\end{array}$ \\
\hline $\begin{array}{l}\text { Yellowtop * } \\
\text { Flaveria linearis }\end{array}$ & $24-48$ & $\begin{array}{l}\text { Full sun to } \\
\text { partial shade }\end{array}$ & Low & $\begin{array}{l}\text { Erect to sprawling perennial with large clusters of tiny yellow } \\
\text { flowers throughout summer. Vigorous and easy to grow, but } \\
\text { cannot tolerate wind exposure. }\end{array}$ \\
\hline $\begin{array}{l}\text { Indian blanket } \\
\text { Gaillardia } \\
\text { pulchella }\end{array}$ & $12-24$ & Full sun & High & $\begin{array}{l}\text { Colorful annual or short-lived perennial. As easily grown as } \\
\text { it is beautiful. Considerable variation in flower color; typical } \\
\text { variety bears red flowers with yellow-tipped petals. Plant in } \\
\text { open site with good drainage. }\end{array}$ \\
\hline $\begin{array}{l}\text { Pineland } \\
\text { heliotrope } \\
\text { Heliotropium } \\
\text { polyphyllum }\end{array}$ & $6-12$ & $\begin{array}{l}\text { Full sun to } \\
\text { partial shade }\end{array}$ & High & $\begin{array}{l}\text { Sparse, partly erect or prostrate perennial composed of thin } \\
\text { stems that arch at the top and bear tiny yellow flowers along } \\
\text { the top side. Slowly forms large patches in cultivation. }\end{array}$ \\
\hline $\begin{array}{l}\text { Pineland lantana } \\
\text { Lantana } \\
\text { depressa }\end{array}$ & $24-48$ & Full sun & High & $\begin{array}{l}\text { Low, sprawling, woody shrub with small, yellow flowers that } \\
\text { attract a variety of butterflies. Beware of the invasive } L \text {. } \\
\text { camara. }\end{array}$ \\
\hline $\begin{array}{l}\text { Pennyroyal } \\
\text { Piloblephis rigida }\end{array}$ & $6-24$ & Full sun & Low & $\begin{array}{l}\text { Low-growing perennial. Not picky about soil, but likes it dry. } \\
\text { Small, shrimp-like flowers emerge from green, scale-like } \\
\text { brachts. Attractive to butterflies. }\end{array}$ \\
\hline
\end{tabular}


Table 1. Native wildflowers for south Florida, listed in alphabetical order by scientific name. (Note: * indicates plants commonly available in native nurseries in south Florida.)

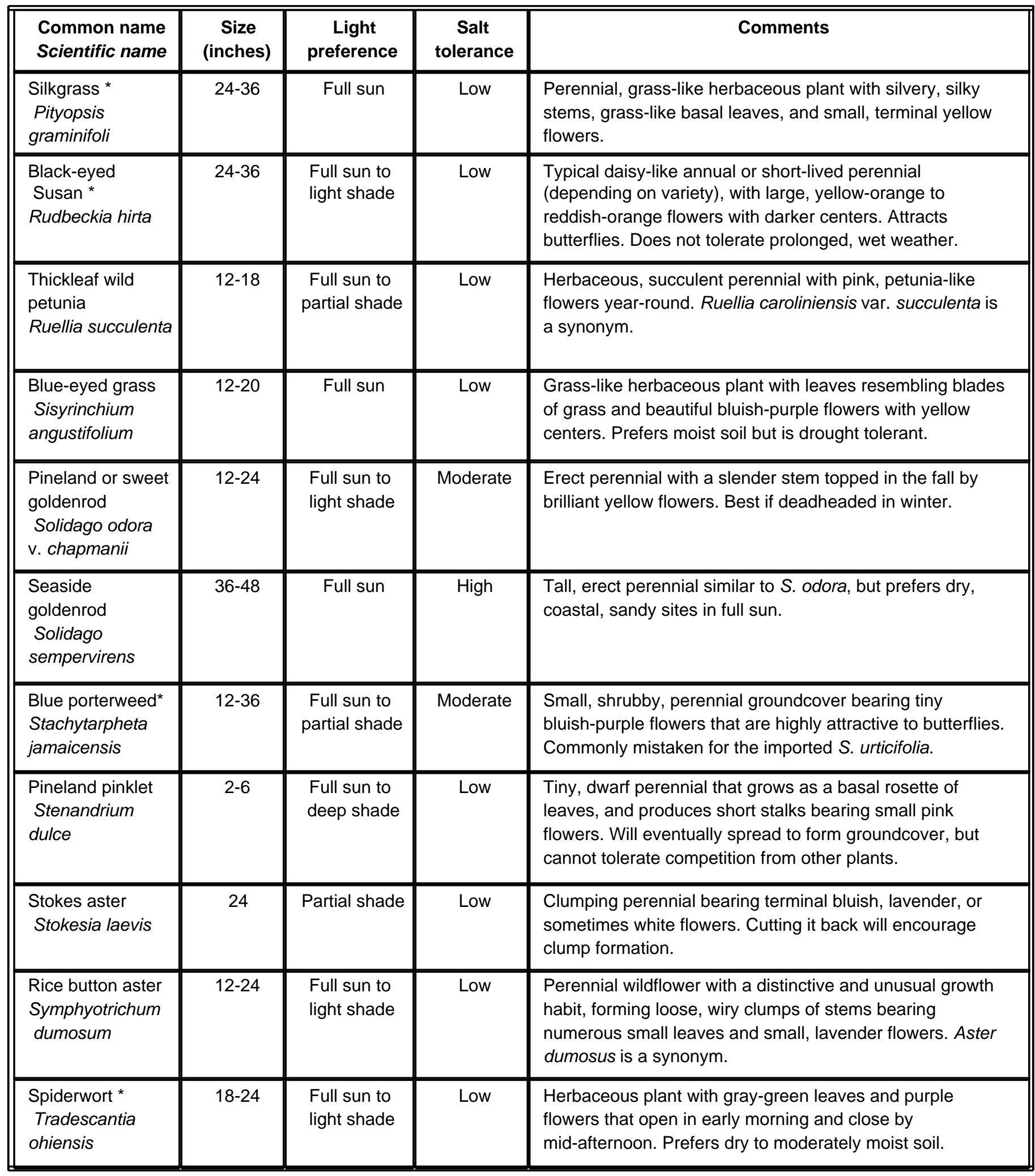


Table 2. Native shrubs and small trees for south Florida, listed in alphabetical order by scientific name. (Note: * indicates plants commonly available in native nurseries in south Florida.)

\begin{tabular}{|c|c|c|c|c|}
\hline $\begin{array}{l}\text { Common name } \\
\text { Scientific name }\end{array}$ & $\begin{array}{c}\text { Size } \\
\text { (feet) }\end{array}$ & $\begin{array}{l}\text { Light } \\
\text { preference }\end{array}$ & $\begin{array}{l}\text { Salt } \\
\text { tolerance }\end{array}$ & Comments \\
\hline $\begin{array}{l}\text { Sweet acacia * } \\
\text { Acacia farnesiana }\end{array}$ & 15 & Full sun & High & $\begin{array}{l}\text { Small tree with sweet-smelling, yellow, "pom-pom-like" } \\
\text { inflorescences. Needs good drainage. }\end{array}$ \\
\hline $\begin{array}{l}\text { Pineland acacia } \\
\text { Acacia pinetorum }\end{array}$ & $4-1$ & Full sun & High & $\begin{array}{l}\text { Dwarf, spiny shrub with delicate, gray to gray-green, } \\
\text { bipinnate leaves and round, "pom-pom-like" inflorescences. } \\
\text { Flowers have strong, sweet fragrance. }\end{array}$ \\
\hline $\begin{array}{l}\text { Torchwood } \\
\text { Amyris elemifera }\end{array}$ & $8-15$ & $\begin{array}{l}\text { Full sun to } \\
\text { partial shade }\end{array}$ & Moderate & $\begin{array}{l}\text { Evergreen, aromatic shrub or small tree. Bark gray brown. } \\
\text { Leaves compound; leaflets dark, shiny green. White flowers } \\
\text { fragrant, followed by purple or black edible fruit. }\end{array}$ \\
\hline $\begin{array}{l}\text { Marlberry * } \\
\text { Ardisia } \\
\text { escallonioides }\end{array}$ & $5-20$ & Partial shade & High & $\begin{array}{l}\text { Shrub to small tree with attractive foliage and berries. Can be } \\
\text { used as small specimen tree or barrier shrub. Attracts birds. } \\
\text { Does poorly in full sun. }\end{array}$ \\
\hline $\begin{array}{l}\text { Beautyberry * } \\
\text { Callicarpa } \\
\text { Americana }\end{array}$ & $6-8$ & $\begin{array}{l}\text { Full sun to } \\
\text { partial shade }\end{array}$ & Moderate & $\begin{array}{l}\text { "Leggy" shrub, especially when grown in shade. Flowers are } \\
\text { insignificant, but the long-lasting purple berries are quite } \\
\text { attractive and provide a good food source for birds. }\end{array}$ \\
\hline $\begin{array}{l}\text { Spicewood } \\
\text { Calyptranthes } \\
\text { pallens }\end{array}$ & $5-25$ & $\begin{array}{l}\text { Partial } \\
\text { Shade }\end{array}$ & Moderate & $\begin{array}{l}\text { Small, shrubby tree with aromatic leaves, burgundy-tinged } \\
\text { new growth, and insignificant white flowers. Can be sheared } \\
\text { and grown as a hedge. Prefers moist soil. }\end{array}$ \\
\hline $\begin{array}{l}\text { Jamaica caper * } \\
\text { Capparis } \\
\text { cynophallophora }\end{array}$ & 18 & $\begin{array}{l}\text { Full sun to } \\
\text { partial shade }\end{array}$ & High & $\begin{array}{l}\text { Slow-growing, undemanding plant with attractive white, } \\
\text { fragrant, spidery, lily-like. With time, can be shaped into } \\
\text { attractive hedge. }\end{array}$ \\
\hline $\begin{array}{l}\text { Cinnamon bark } \\
\text { Canella winterana }\end{array}$ & $10-30$ & $\begin{array}{l}\text { Full sun to } \\
\text { light shade }\end{array}$ & High & $\begin{array}{l}\text { Very attractive, small, slow-growing tree with dense, broad } \\
\text { crown; thick, aromatic leaves; fragrant, purplish flowers; and } \\
\text { red berries. Flower aroma resembles daffodils; inner bark } \\
\text { aroma resembles cinnamon. All parts poisonous, except } \\
\text { berries. Canella alba is a synonym. }\end{array}$ \\
\hline $\begin{array}{l}\text { Limber caper * } \\
\text { Capparis flexuosa }\end{array}$ & $12-25$ & $\begin{array}{l}\text { Full sun to } \\
\text { full shade }\end{array}$ & High & $\begin{array}{l}\text { Medium to large vining shrub with large, fragrant flowers in } \\
\text { spring and beanlike fruit capsules in summer or fall. }\end{array}$ \\
\hline $\begin{array}{l}\text { Bird pepper } \\
\text { Capsicum annuum } \\
\text { v. glabriusculum }\end{array}$ & $1.5-3$ & $\begin{array}{l}\text { Full sun to } \\
\text { deep shade }\end{array}$ & Low & $\begin{array}{l}\text { Dwarf, dense, evergreen shrub with glossy, dark green } \\
\text { leaves and tiny chile peppers that are highly attractive to } \\
\text { birds. Wild ancestor from which our common sweet and chile } \\
\text { peppers were derived. }\end{array}$ \\
\hline $\begin{array}{l}\text { Cocoplum * } \\
\text { Chrysobalanus } \\
\text { icaco }\end{array}$ & $3-15$ & $\begin{array}{l}\text { Full sun to } \\
\text { partial shade }\end{array}$ & High & $\begin{array}{l}\text { Shrub to small tree with attractive red new foliage. Often } \\
\text { used as hedge, but will thin if planted in too much shade. } \\
\text { Spreading and erect forms are available. }\end{array}$ \\
\hline $\begin{array}{l}\text { Snowberry } \\
\text { Chiococca alba }\end{array}$ & $2-3$ & Full sun & Low & $\begin{array}{l}\text { Vining shrub with glossy green, elliptical to lanceolate leaves, } \\
\text { and tiny white flowers. Chiococca parvifolia is a synonym. }\end{array}$ \\
\hline $\begin{array}{l}\text { Fiddlewood* } \\
\text { Citharexylum } \\
\text { spinosum }\end{array}$ & $12-30$ & $\begin{array}{l}\text { Full sun to } \\
\text { partial shade }\end{array}$ & High & $\begin{array}{l}\text { Large, densely leafy shrub to small tree with glossy, elliptical } \\
\text { leaves and small, white, fragrant flowers. Female plants bear } \\
\text { orange-brown berries that attract birds and other wildlife. } \\
\text { Citharexylum fruticosum is a synonym. }\end{array}$ \\
\hline
\end{tabular}


Table 2. Native shrubs and small trees for south Florida, listed in alphabetical order by scientific name. (Note: * indicates plants commonly available in native nurseries in south Florida.)

\begin{tabular}{|c|c|c|c|c|}
\hline $\begin{array}{l}\text { Common name } \\
\text { Scientific name }\end{array}$ & $\begin{array}{l}\text { Size } \\
\text { (feet) }\end{array}$ & $\begin{array}{l}\text { Light } \\
\text { preference }\end{array}$ & $\begin{array}{l}\text { Salt } \\
\text { tolerance }\end{array}$ & Comments \\
\hline $\begin{array}{l}\text { Pigeon plum * } \\
\text { Coccoloba } \\
\text { diversifolia }\end{array}$ & $5-30$ & $\begin{array}{l}\text { Full sun to } \\
\text { partial shade }\end{array}$ & High & $\begin{array}{l}\text { Large shrub to small tree with dense, narrowly rounded, } \\
\text { evergreen crown and attractive, peeling bark. Small, dark } \\
\text { purple berries on female trees attract birds. Susceptible to } \\
\text { weevils. }\end{array}$ \\
\hline $\begin{array}{l}\text { Sea grape * } \\
\text { Coccoloba uvifera }\end{array}$ & $10-50$ & Full sun & High & $\begin{array}{l}\text { Large shrub to large tree with large, thick, saucer-like leaves } \\
\text { bearing attractive venation and edible fruit. Leaves can be } \\
\text { messy. Susceptible to weevils. }\end{array}$ \\
\hline $\begin{array}{l}\text { Coffee colubrina * } \\
\text { Colubrina } \\
\text { arborescens }\end{array}$ & 20 & $\begin{array}{l}\text { Full sun to } \\
\text { light shade }\end{array}$ & High & $\begin{array}{l}\text { Large, coarse-leaved shrub/small tree with open crown of } \\
\text { large, tri-lobed leaves, inconspicuous green flowers, and } \\
\text { 3-seeded capsules. Attractive to butterflies and many other } \\
\text { beneficial insects. }\end{array}$ \\
\hline $\begin{array}{l}\text { Buttonwood * } \\
\text { Conocarpus } \\
\text { erectus }\end{array}$ & $5-50$ & $\begin{array}{l}\text { Full sun to } \\
\text { partial shade }\end{array}$ & High & $\begin{array}{l}\text { Both green and silver leaved forms available; the latter is } \\
\text { more attractive but also more susceptible to sooty mold and } \\
\text { insect problems. Insignificant flowers are followed by small, } \\
\text { button-like seed pods. Susceptible to some pests. Do not } \\
\text { plant in marl soil. }\end{array}$ \\
\hline $\begin{array}{l}\text { Bloodberry } \\
\text { Cordia globosa }\end{array}$ & $4-9$ & $\begin{array}{l}\text { Full sun to } \\
\text { moderate } \\
\text { shade }\end{array}$ & Low & $\begin{array}{l}\text { Small, densely branched, evergreen shrub often growing in a } \\
\text { rounded, symmetrical shape. Tiny white flowers are followed } \\
\text { by small, brilliant red berries. Makes a great hedge, as it can } \\
\text { be trimmed to nearly any shape or size. }\end{array}$ \\
\hline $\begin{array}{l}\text { Rhacoma } \\
\text { Crossopetalum } \\
\text { rhacoma }\end{array}$ & $1.5-6$ & $\begin{array}{l}\text { Full sun to } \\
\text { moderate } \\
\text { shade }\end{array}$ & High & $\begin{array}{l}\text { Different forms available, from low and spreading to erect } \\
\text { and shrub-like. All possess tiny leaves and bright red berries. }\end{array}$ \\
\hline $\begin{array}{l}\text { Pepperbush } \\
\text { Croton humilis }\end{array}$ & 3 & $\begin{array}{l}\text { Full sun to } \\
\text { moderate } \\
\text { shade }\end{array}$ & Low & $\begin{array}{l}\text { Dwarf, rounded shrub with large, dark green leaves bearing } \\
\text { numerous tiny, white, star-shaped hairs. Flowers form white, } \\
\text { conspicuous starbursts. }\end{array}$ \\
\hline $\begin{array}{l}\text { Varnish leaf * } \\
\text { Dodonaea viscosa }\end{array}$ & 18 & $\begin{array}{l}\text { Full sun to } \\
\text { partial shade }\end{array}$ & High & $\begin{array}{l}\text { Grown for its attractive stiff, shiny green leaves. Showy, } \\
\text { yellowish, 3-winged capsules produced in terminal clusters; } \\
\text { brown, pink or purple at maturity. Dense and fast growing. } \\
\text { Used as free-standing specimen or hedge. Excellent for } \\
\text { beach-front. }\end{array}$ \\
\hline $\begin{array}{l}\text { Coral bean * } \\
\text { Erythrina herbacea }\end{array}$ & 3-15 & Full sun & Low & $\begin{array}{l}\text { Deciduous, 3-lobed compound leaves. Showy scarlet } \\
\text { blossoms on tall stalks in spring, followed by large beans that } \\
\text { split to reveal bright red seeds. May grow into small tree. All } \\
\text { parts of this plant are poisonous; twigs prickly. }\end{array}$ \\
\hline $\begin{array}{l}\text { White stopper * } \\
\text { Eugenia axillaris }\end{array}$ & $5-20$ & $\begin{array}{c}\text { Full sun to } \\
\text { dense shade }\end{array}$ & High & $\begin{array}{l}\text { Pale, whitish bark and aromatic foliage that can be } \\
\text { overpowering. Small, white flowers in midsummer are } \\
\text { followed by small, purplish berries. Attractive to birds. }\end{array}$ \\
\hline $\begin{array}{l}\text { Redberry stopper } \\
\text { Eugenia confusa }\end{array}$ & $6-18$ & $\begin{array}{l}\text { Full sun to } \\
\text { partial shade }\end{array}$ & High & $\begin{array}{l}\text { Slow-growing shrub to small tree with attractive glossy } \\
\text { leaves and red berries. Used as specimen plant or hedge. } \\
\text { Narrow, upright growth suitable for restricted site. }\end{array}$ \\
\hline
\end{tabular}


Table 2. Native shrubs and small trees for south Florida, listed in alphabetical order by scientific name. (Note: * indicates plants commonly available in native nurseries in south Florida.)

\begin{tabular}{|c|c|c|c|c|}
\hline $\begin{array}{l}\text { Common name } \\
\text { Scientific name }\end{array}$ & $\begin{array}{c}\text { Size } \\
\text { (feet) }\end{array}$ & $\begin{array}{l}\text { Light } \\
\text { preference }\end{array}$ & $\begin{array}{l}\text { Salt } \\
\text { tolerance }\end{array}$ & Comments \\
\hline $\begin{array}{l}\text { Spanish stopper } \\
\text { * } \\
\text { Eugenia foetida }\end{array}$ & $18-36$ & $\begin{array}{l}\text { Full sun to } \\
\text { shade }\end{array}$ & High & $\begin{array}{l}\text { Large shrub or small tree densely clothed with small, rounded } \\
\text { leaves. Smallest flowers and fruits of all stoppers, but still } \\
\text { attractive to wildlife. Excellent foundation or specimen plant. }\end{array}$ \\
\hline $\begin{array}{l}\text { Red stopper } \\
\text { Eugenia rhombea }\end{array}$ & 9 & $\begin{array}{l}\text { Full sun to } \\
\text { shade }\end{array}$ & High & $\begin{array}{l}\text { Reddish brown bark and elegant growth habit. Branches } \\
\text { produced in flat sprays perpendicular to main trunk. } \\
\text { Slow-growing and does not flower or fruit as a young plant. }\end{array}$ \\
\hline $\begin{array}{l}\text { Inkwood } \\
\text { Exothea paniculata }\end{array}$ & $10-30$ & $\begin{array}{l}\text { Full sun to } \\
\text { partial shade }\end{array}$ & Moderate & $\begin{array}{l}\text { Evergreen shrub to medium tree with narrow, erect growth } \\
\text { habit. Bark gray. Leaves shiny dark green. Small clusters of } \\
\text { insignificant, fragrant, white flowers. Wood very strong. Sap } \\
\text { turns black when exposed to air. }\end{array}$ \\
\hline $\begin{array}{l}\text { Florida privet } \\
\text { Forestiera } \\
\text { segregata }\end{array}$ & 10 & Full sun & High & $\begin{array}{l}\text { Bushy shrub with insignificant flowers. Wildlife attracted to its } \\
\text { black fruit. Very tolerant of alkaline soils. Can be used as } \\
\text { hedge plant in place of ligustrum. }\end{array}$ \\
\hline $\begin{array}{l}\text { Lignum vitae * } \\
\text { Guaiacum sanctum }\end{array}$ & $6-25$ & Full sun & High & $\begin{array}{l}\text { Attractive, slow-growing, large shrub to small tree, with blue } \\
\text { flowers year-round, mostly in spring. Flowers followed by } \\
\text { yellow seed pods which pop open to reveal shiny, bright red } \\
\text { seeds. Extremely dense, prized wood. }\end{array}$ \\
\hline $\begin{array}{l}\text { Firebush * } \\
\text { Hamelia patens }\end{array}$ & $5-15$ & $\begin{array}{l}\text { Shady to } \\
\text { dappled sun }\end{array}$ & Low & $\begin{array}{l}\text { Produces attractive orange/red flowers year-round. } \\
\text { Indifferent to soil, providing drainage is good. In shade, } \\
\text { becomes less shrubby and more tree-like. Highly attractive to } \\
\text { butterflies. }\end{array}$ \\
\hline $\begin{array}{l}\text { Joewood } \\
\text { Jacquinia keyensis }\end{array}$ & 10 & $\begin{array}{l}\text { Full sun to } \\
\text { partial shade }\end{array}$ & High & $\begin{array}{l}\text { Slow-growing shrub with attractive foliage and showy, } \\
\text { fragrant blooms. Good resistance to wind and salt spray. } \\
\text { Shade from hottest sun beneficial. All parts poisonous. }\end{array}$ \\
\hline $\begin{array}{l}\text { Pineland lantana } \\
\text { Lantana depressa }\end{array}$ & $2-4$ & Full sun & High & See description in Table 1 above. \\
\hline $\begin{array}{l}\text { Buttonsage } \\
\text { Lantana } \\
\text { involucrata }\end{array}$ & $4-5$ & Full sun & High & $\begin{array}{l}\text { Upright, woody shrub with small, gray-green, rough-textured } \\
\text { leaves and small white flowers. }\end{array}$ \\
\hline $\begin{array}{l}\text { Morinda, redgal } \\
\text { Morinda royoc }\end{array}$ & $5-10$ & $\begin{array}{l}\text { Full sun to } \\
\text { partial shade }\end{array}$ & High & $\begin{array}{l}\text { Evergreen, vine-like shrub with long, thin leaves, small } \\
\text { whitish to reddish flowers, and distinct yellowish fruit that } \\
\text { smells of cheese when bruised. }\end{array}$ \\
\hline $\begin{array}{l}\text { Long-stalked } \\
\text { stopper } \\
\text { Mosiera longipes }\end{array}$ & $1.5-3$ & $\begin{array}{l}\text { Full sun to } \\
\text { light shade }\end{array}$ & Low & $\begin{array}{l}\text { Small shrub with small, dark green, glossy leaves and pale } \\
\text { green new growth. Small white flowers are delicate in } \\
\text { appearance, and are followed by blackish purple berries. } \\
\text { Psidium longipes is a synonym. }\end{array}$ \\
\hline $\begin{array}{l}\text { Simpson's stopper } \\
\text { Myrcianthes } \\
\text { fragrans }\end{array}$ & $6-20$ & $\begin{array}{l}\text { Full sun to } \\
\text { partial shade }\end{array}$ & High & $\begin{array}{l}\text { One of the most beautiful and ornamental native woody } \\
\text { plants. Densely branched, densely leafy large shrub to small } \\
\text { tree. Pure white, puffy flowers followed by large, bright } \\
\text { orange berries that attract birds. }\end{array}$ \\
\hline
\end{tabular}


Table 2. Native shrubs and small trees for south Florida, listed in alphabetical order by scientific name. (Note: * indicates plants commonly available in native nurseries in south Florida.)

\begin{tabular}{|c|c|c|c|c|}
\hline $\begin{array}{l}\text { Common name } \\
\text { Scientific name }\end{array}$ & $\begin{array}{l}\text { Size } \\
\text { (feet) }\end{array}$ & $\begin{array}{l}\text { Light } \\
\text { preference }\end{array}$ & $\begin{array}{c}\text { Salt } \\
\text { tolerance }\end{array}$ & Comments \\
\hline $\begin{array}{l}\text { Wax myrtle * } \\
\text { Myrica cerifera }\end{array}$ & $10-25$ & $\begin{array}{l}\text { Full sun to } \\
\text { partial shade }\end{array}$ & Low & $\begin{array}{l}\text { Fast-growing shrub to small tree with small, evergreen } \\
\text { leaves, inconspicuous flowers, and waxy gray fruit attached } \\
\text { to the twigs. Leaves and fruit smell like bayberry. Tolerates } \\
\text { periodic flooding. Natural insect repellant. Attracts birds. Tip } \\
\text { dieback can be a problem. }\end{array}$ \\
\hline $\begin{array}{l}\text { Lancewood } \\
\text { Ocotea coriacea }\end{array}$ & $15-25$ & $\begin{array}{l}\text { Full sun to } \\
\text { full shade }\end{array}$ & Low & $\begin{array}{l}\text { Relatively short-lived, densely branched shrub or small tree } \\
\text { with broad, oval crown, glossy leaves, inconspicuous } \\
\text { flowers, and distinctive, dark purple or black berries held in a } \\
\text { yellowish or reddish cup. Nectandra coriacea is a synonym. }\end{array}$ \\
\hline $\begin{array}{l}\text { Bitterbush } \\
\text { Picramnia } \\
\text { pentandra }\end{array}$ & $5-10$ & $\begin{array}{l}\text { Full sun to } \\
\text { deep shade }\end{array}$ & Low & $\begin{array}{l}\text { Tough, shrubby tree with compound leaves and long, } \\
\text { drooping inflorescences bearing tiny, fragrant flowers. Fruit } \\
\text { are eaten by a variety of animals. Also the larval food plant } \\
\text { of the bush sulphur butterfly. }\end{array}$ \\
\hline $\begin{array}{l}\text { Blackbead * } \\
\text { Pithecellobium } \\
\text { keyense }\end{array}$ & $10-20$ & $\begin{array}{l}\text { Full sun to } \\
\text { partial shade }\end{array}$ & High & $\begin{array}{l}\text { Large shrub or small tree with leaves divided into four } \\
\text { leaflets. New leaves tinged with red or maroon. Flowers are } \\
\text { delicate, fragrant, and white to pink. Contorted bean pods } \\
\text { split open to reveal black seeds covered with a fleshy red aril. } \\
\text { Wood is weak. }\end{array}$ \\
\hline $\begin{array}{l}\text { Bahama or } \\
\text { privet-leaf wild } \\
\text { coffee } \\
\text { Psychotria } \\
\text { ligustrifolia }\end{array}$ & $6-9$ & $\begin{array}{l}\text { Partial to full } \\
\text { shade }\end{array}$ & Low & $\begin{array}{l}\text { Densely leafy large shrub or small tree with dark green, } \\
\text { glossy foliage. Like the other native wild coffees, it also } \\
\text { bears a proliferation of red berries. Psychotria bahamensis is } \\
\text { a synonym. }\end{array}$ \\
\hline $\begin{array}{l}\text { Wild coffee * } \\
\text { Psychotria nervosa }\end{array}$ & $6-9$ & $\begin{array}{l}\text { Partial to full } \\
\text { shade }\end{array}$ & Low & $\begin{array}{l}\text { Small, understory shrub with distinctive glossy, dark green } \\
\text { leaves with deeply impressed side veins. Bears small, } \\
\text { insignificant flowers, followed by prolific red berries. }\end{array}$ \\
\hline $\begin{array}{l}\text { Velvetleaf wild } \\
\text { coffee * }^{*} \\
\text { Psychotria sulzneri }\end{array}$ & $6-9$ & $\begin{array}{l}\text { Partial to full } \\
\text { shade }\end{array}$ & Low & $\begin{array}{l}\text { Similar in all respects to } P \text {. nervosa, but with velvety, deep } \\
\text { blue-green foliage. All wild coffees require some shade to } \\
\text { look their best. }\end{array}$ \\
\hline $\begin{array}{l}\text { White indigoberry } \\
\text { Randia aculeata }\end{array}$ & $1.5-10$ & $\begin{array}{l}\text { Full sun to } \\
\text { partial shade }\end{array}$ & High & $\begin{array}{l}\text { Non-descript, upright shrub with fragrant flowers. Female } \\
\text { plants bear white berries that are an intense blue inside. Main } \\
\text { attribute is ability to grow under adverse conditions. }\end{array}$ \\
\hline $\begin{array}{l}\text { Myrsine } \\
\text { Rapanea punctata }\end{array}$ & 25 & $\begin{array}{l}\text { Full sun to } \\
\text { partial shade }\end{array}$ & Low & $\begin{array}{l}\text { Evergreen large shrub to small tree resembling marlberry. } \\
\text { Bark pale gray. Flowers small, white, sometimes with some } \\
\text { purple. Berries dark purple or black. Myrsine floridana is a } \\
\text { synonym. }\end{array}$ \\
\hline $\begin{array}{l}\text { Rouge plant } \\
\text { Rivina humilis }\end{array}$ & $3-5$ & $\begin{array}{l}\text { Full sun to } \\
\text { full shade }\end{array}$ & Low & $\begin{array}{l}\text { Unassuming, small, carefree plant with dark green, glossy } \\
\text { leaves, tiny white flowers, and bright red berries. }\end{array}$ \\
\hline $\begin{array}{l}\text { American } \\
\text { elderberry } \\
\text { Sambucus } \\
\text { canadensis }\end{array}$ & $10-15$ & $\begin{array}{l}\text { Full sun to } \\
\text { partial shade }\end{array}$ & Low & $\begin{array}{l}\text { Bushy, multi-stemmed shrub with deciduous, compound } \\
\text { leaves, tiny, star-shaped, white flowers, and shiny, } \\
\text { blue-black fruit. Provides colorful autumn display of yellows, } \\
\text { oranges, and reds. Branches brittle. Forms dense thickets by } \\
\text { suckering from roots. }\end{array}$ \\
\hline
\end{tabular}


Table 2. Native shrubs and small trees for south Florida, listed in alphabetical order by scientific name. (Note: * indicates plants commonly available in native nurseries in south Florida.)

\begin{tabular}{|c|c|c|c|c|}
\hline $\begin{array}{l}\text { Common name } \\
\text { Scientific name }\end{array}$ & $\begin{array}{c}\text { Size } \\
\text { (feet) }\end{array}$ & $\begin{array}{l}\text { Light } \\
\text { preference }\end{array}$ & $\begin{array}{l}\text { Salt } \\
\text { tolerance }\end{array}$ & Comments \\
\hline $\begin{array}{l}\text { Maidenbush } \\
\text { Savia bahamensis }\end{array}$ & $10-15$ & Partial shade & High & $\begin{array}{l}\text { Shrub or small tree with whitish bark, pale green, alternate } \\
\text { leaves. Native to Florida Keys. }\end{array}$ \\
\hline $\begin{array}{l}\text { Inkberry } \\
\text { Scaevola plumieri }\end{array}$ & $2-4$ & $\begin{array}{l}\text { Full sun to } \\
\text { partial shade }\end{array}$ & High & $\begin{array}{l}\text { Short plant with succulent leaves and insignificant pink and } \\
\text { white flowers. Spreads by underground rhizomes. } \\
\text { Well-suited to sandy soils at beach-front. }\end{array}$ \\
\hline $\begin{array}{l}\text { Florida boxwood } \\
\text { Schaefferia } \\
\text { frutescens }\end{array}$ & $10-30$ & $\begin{array}{l}\text { Partial to full } \\
\text { shade }\end{array}$ & Low & $\begin{array}{l}\text { Thin-branched, leafy shrub to small tree with dark green, } \\
\text { shiny leaves and flowers and fruit (on female plants) all year. } \\
\text { Can be pruned as a hedge. }\end{array}$ \\
\hline $\begin{array}{l}\text { Bahama senna * } \\
\text { Senna mexicana } \\
\text { var. chapmanii }\end{array}$ & $3-5$ & $\begin{array}{l}\text { Full sun to } \\
\text { partial shade }\end{array}$ & High & $\begin{array}{l}\text { Upright or sprawling shrub, with bipinnate leaves and terminal } \\
\text { clusters of beautiful, orange-red flowers. }\end{array}$ \\
\hline $\begin{array}{l}\text { Saw palmetto * } \\
\text { Serenoa repens }\end{array}$ & $3-8$ & $\begin{array}{l}\text { Full sun to } \\
\text { partial shade }\end{array}$ & High & $\begin{array}{l}\text { Clumping fan palm with prostrate or upright trunks. One of } \\
\text { the most abundant native palms in Florida. Green and silver } \\
\text { forms available. Spreading tendency can be a problem when } \\
\text { left uncontrolled. Petioles heavily armed. }\end{array}$ \\
\hline $\begin{array}{l}\text { Willow bustic * } \\
\text { Sideroxylon } \\
\text { salicifolium }\end{array}$ & $10-30$ & $\begin{array}{l}\text { Full sun to } \\
\text { partial shade }\end{array}$ & Low & $\begin{array}{l}\text { Evergreen woody shrub or medium tree. Bark gray. Leaves } \\
\text { medium green, shiny above and dull below, with yellow veins. } \\
\text { Flowers produced from warty, pod-like structures. }\end{array}$ \\
\hline $\begin{array}{l}\text { Necklace-pod* } \\
\text { Sophora } \\
\text { tomentosa }\end{array}$ & 6 & Full sun & High & $\begin{array}{l}\text { Large, densely branched shrub with natural rounded shape. } \\
\text { Bears clusters of yellow flowers at tips of branches. } \\
\text { Fast-growing and easily cultivated. Seeds poisonous. }\end{array}$ \\
\hline $\begin{array}{l}\text { Bay cedar } \\
\text { Suriana maritima }\end{array}$ & $6-12$ & Full sun & High & $\begin{array}{l}\text { Evergreen shrub or small tree with clusters of small, leathery } \\
\text { leaves and attractive, peeling bark. Will grow in sand or on } \\
\text { bare rock. Good choice for beach-front sites. }\end{array}$ \\
\hline $\begin{array}{l}\text { Tetrazygia, West } \\
\text { Indian lilac } \\
\text { Tetrazygia bicolor }\end{array}$ & $6-12$ & $\begin{array}{l}\text { Full sun to } \\
\text { light shade }\end{array}$ & Low & $\begin{array}{l}\text { Extremely ornamental shrub or small tree with elegant, } \\
\text { glossy, dark green foliage and beautiful white and yellow } \\
\text { flowers followed by purple-black berries. Highly attractive to } \\
\text { birds. Branches die when pruned. }\end{array}$ \\
\hline $\begin{array}{l}\text { Florida trema } \\
\text { Trema micranthum }\end{array}$ & $5-30$ & $\begin{array}{l}\text { Full sun to } \\
\text { partial shade }\end{array}$ & Low & $\begin{array}{l}\text { Usually an evergreen, sprawling shrub, but can grow to } \\
\text { medium sized tree. Leaves alternate on branch, dull green, } \\
\text { and rough textured. Insignificant flowers followed by tiny, } \\
\text { yellow-orange berries all along the smallest branches. }\end{array}$ \\
\hline $\begin{array}{l}\text { Spanish bayonet * } \\
\text { Yucca aloifolia }\end{array}$ & $5-20$ & $\begin{array}{l}\text { Full sun or } \\
\text { partial shade }\end{array}$ & High & $\begin{array}{l}\text { Trunk-forming yucca with dangerously pointed, strap-like } \\
\text { leaves. Trunks often topped with large, upright clusters of } \\
\text { creamy white flowers. Spreads to form thicket. }\end{array}$ \\
\hline $\begin{array}{l}\text { Bear grass, } \\
\text { Adamneedle } \\
\text { Yucca filamentosa }\end{array}$ & $3-6$ & Full sun & High & $\begin{array}{l}\text { Long, green, spear-like leaves edged with white threads, } \\
\text { forming a basal rosette. White, bell-shaped flowers bloom in } \\
\text { terminal spikes from mid to late summer. }\end{array}$ \\
\hline
\end{tabular}


Table 2. Native shrubs and small trees for south Florida, listed in alphabetical order by scientific name. (Note: * indicates plants commonly available in native nurseries in south Florida.)

\begin{tabular}{||l|c|c|c|l||}
\hline \hline $\begin{array}{l}\text { Common name } \\
\text { Scientific name }\end{array}$ & $\begin{array}{c}\text { Size } \\
\text { (feet) }\end{array}$ & $\begin{array}{c}\text { Light } \\
\text { preference }\end{array}$ & $\begin{array}{c}\text { Salt } \\
\text { tolerance }\end{array}$ & \multicolumn{1}{c||}{ Comments } \\
\hline $\begin{array}{l}\text { Florida coontie * } \\
\text { Zamia floridana }\end{array}$ & $1-5$ & $\begin{array}{c}\text { Full sun to } \\
\text { shade }\end{array}$ & High & $\begin{array}{l}\text { Florida's only native cycad. Comes in a variety of sizes and } \\
\text { with narrow to wide leaflets. Separate male and female } \\
\text { plants. Sole larval food source for atala hairstreak butterfly. } \\
\text { Requires well-drained soil. Zamia pumila and Z. integrifolia } \\
\text { are synonyms. }\end{array}$ \\
\hline $\begin{array}{l}\text { Wild lime * } \\
\text { Zanthoxylum } \\
\text { agara }\end{array}$ & 20 & Full sun & Moderate & $\begin{array}{l}\text { Attractive tree with recurved spines, lime-scented foliage, } \\
\text { and insignificant yellow flowers that attract butterflies. }\end{array}$ \\
\hline \hline
\end{tabular}

Table 3. Native medium and large trees for south Florida, listed in alphabetical order by scientific name. (Note: * indicates plants commonly available in native nurseries in south Florida.)

\begin{tabular}{|c|c|c|c|c|}
\hline $\begin{array}{l}\text { Common name } \\
\text { Scientific name }\end{array}$ & $\begin{array}{l}\text { Size } \\
\text { (feet) }\end{array}$ & $\begin{array}{l}\text { Light } \\
\text { preference }\end{array}$ & $\begin{array}{c}\text { Salt } \\
\text { tolerance }\end{array}$ & Comments \\
\hline $\begin{array}{l}\text { Spineless acacia, } \\
\text { cinnecord * } \\
\text { Acacia } \\
\text { choriophylla }\end{array}$ & 30 & Full sun & High & $\begin{array}{l}\text { Medium-sized tree with dense, rounded crown, long, } \\
\text { bipinnate leaves, and tiny yellow to golden "pom-pom-like" } \\
\text { inflorescences. Spines are much reduced, but still present. } \\
\text { Also mistakenly known as } A \text {. choriophylloides. }\end{array}$ \\
\hline $\begin{array}{l}\text { Strongback * } \\
\text { Bourreria } \\
\text { succulenta }\end{array}$ & 30 & $\begin{array}{l}\text { Full sun to } \\
\text { partial shade }\end{array}$ & High & $\begin{array}{l}\text { Rapidly growing, evergreen tree with numerous small, } \\
\text { white flowers followed by orange berries that attract birds. } \\
\text { Commonly -- but mistakenly -- known as strongbark. }\end{array}$ \\
\hline $\begin{array}{l}\text { Gumbo limbo * } \\
\text { Bursera simaruba }\end{array}$ & $20-50$ & $\begin{array}{l}\text { Full sun to } \\
\text { partial shade }\end{array}$ & Moderate & $\begin{array}{l}\text { Grown for its attractive, peeling, bronze to red bark and its } \\
\text { large, appealing form. Cuttings take easily but are } \\
\text { weak-rooted. Not a strong tree; limbs may break in strong } \\
\text { winds. }\end{array}$ \\
\hline $\begin{array}{l}\text { Myrtle-of-the- } \\
\text { River } \\
\text { Calyptranthes } \\
\text { zuzygium }\end{array}$ & $20-40$ & $\begin{array}{l}\text { Full sun to } \\
\text { partial shade }\end{array}$ & Moderate & $\begin{array}{l}\text { Large shrub to medium tree with olive-green leaves, pale, } \\
\text { pink-tinged new growth and showy, fragrant, white flowers } \\
\text { Endangered in Florida. Related to spicewood, but with } \\
\text { larger flowers. }\end{array}$ \\
\hline $\begin{array}{l}\text { Cinnamon bark } \\
\text { Canella winterana }\end{array}$ & $10-30$ & $\begin{array}{l}\text { Full sun to } \\
\text { light shade }\end{array}$ & High & See description in Table 2 above. \\
\hline $\begin{array}{l}\text { Satinleaf * } \\
\text { Chrysophyllum } \\
\text { oliviforme }\end{array}$ & 30 & Full sun & Moderate & $\begin{array}{l}\text { Very attractive tree. Tops of leaves glossy, vivid green; } \\
\text { underside covered with soft, coppery brown hairs. Can be } \\
\text { difficult to establish. }\end{array}$ \\
\hline $\begin{array}{l}\text { Fiddlewood * } \\
\text { Citharexylum } \\
\text { spinosum }\end{array}$ & $12-30$ & $\begin{array}{l}\text { Full sun to } \\
\text { partial shade }\end{array}$ & High & See description in Table 2 above. \\
\hline $\begin{array}{l}\text { Pigeon plum * } \\
\text { Coccoloba } \\
\text { diversifolia }\end{array}$ & $5-30$ & $\begin{array}{l}\text { Full sun to } \\
\text { partial shade }\end{array}$ & High & See description in Table 2 above. \\
\hline
\end{tabular}


Table 3. Native medium and large trees for south Florida, listed in alphabetical order by scientific name. (Note: * indicates plants commonly available in native nurseries in south Florida.)

\begin{tabular}{|c|c|c|c|c|}
\hline $\begin{array}{l}\text { Common name } \\
\text { Scientific name }\end{array}$ & $\begin{array}{l}\text { Size } \\
\text { (feet) }\end{array}$ & $\begin{array}{l}\text { Light } \\
\text { preference }\end{array}$ & $\begin{array}{c}\text { Salt } \\
\text { tolerance }\end{array}$ & Comments \\
\hline $\begin{array}{l}\text { Sea grape * } \\
\text { Coccoloba uvifera }\end{array}$ & $5-50$ & Full sun & High & See description in Table 2 above. \\
\hline $\begin{array}{l}\text { Buttonwood * } \\
\text { Conocarpus } \\
\text { erectus }\end{array}$ & $5-50$ & $\begin{array}{l}\text { Full sun to } \\
\text { partial shade }\end{array}$ & High & See description in Table 2 above. \\
\hline $\begin{array}{l}\text { American } \\
\text { persimmon } \\
\text { Diospyros } \\
\text { virginiana }\end{array}$ & 50 & Full sun & None & $\begin{array}{l}\text { Slow-growing, dioecious, deciduous tree with elliptical, } \\
\text { two-tone leaves and black, textured bark. Females } \\
\text { produce two fruits that ripen to deliciously sweet. Tends to } \\
\text { root sucker. Choose named cultivars, such as 'Triumph'. } \\
\text { Prefers moist soil when in fruit. Attracts wildlife. }\end{array}$ \\
\hline $\begin{array}{l}\text { Inkwood } \\
\text { Exothea } \\
\text { paniculata }\end{array}$ & $10-30$ & $\begin{array}{l}\text { Full sun to } \\
\text { partial shade }\end{array}$ & Moderate & See description in Table 2 above. \\
\hline $\begin{array}{l}\text { Shortleaf fig } \\
\text { Ficus citrifolia }\end{array}$ & $25-50$ & $\begin{array}{l}\text { Full sun to } \\
\text { partial shade }\end{array}$ & Low & $\begin{array}{l}\text { Medium-sized, fast-growing, attractive tree. Lacks typical } \\
\text { aerial roots, but still requires adequate room for root } \\
\text { development. Also known as wild banyan tree. }\end{array}$ \\
\hline $\begin{array}{l}\text { Longleaf blolly } \\
\text { Guapira discolor }\end{array}$ & 30 & $\begin{array}{l}\text { Full sun to } \\
\text { light shade }\end{array}$ & High & $\begin{array}{l}\text { Attractive shade tree, especially for beach-front. Hardy and } \\
\text { adaptable. Greenish flowers insignificant. Female trees } \\
\text { bear small, red berries. }\end{array}$ \\
\hline $\begin{array}{l}\text { Krug holly * } \\
\text { Ilex krugiana }\end{array}$ & 30 & Partial shade & High & $\begin{array}{l}\text { Tropical holly with glossy foliage and black fruits. Well } \\
\text { adapted to south Florida. Small, red berries ripen to black. }\end{array}$ \\
\hline $\begin{array}{l}\text { Black ironwood* } \\
\text { Krugiodendron } \\
\text { ferreum }\end{array}$ & 30 & $\begin{array}{l}\text { Full sun to } \\
\text { light shade }\end{array}$ & Low & $\begin{array}{l}\text { Evergreen tree with dark, emerald green, glossy leaves } \\
\text { and small black berries. Narrow crown allows it to be } \\
\text { planted in tight spaces. }\end{array}$ \\
\hline $\begin{array}{l}\text { Wild tamarind * } \\
\text { Lysiloma } \\
\text { latisiliquum }\end{array}$ & $40-60$ & Full Sun & Moderate & $\begin{array}{l}\text { Attractive, fast-growing tree, but with weak wood. } \\
\text { Bipinnately compound leaves, small, white/pink } \\
\text { inflorescences, and long brown seed pods. }\end{array}$ \\
\hline $\begin{array}{l}\text { Red mulberry } \\
\text { Morus rubra }\end{array}$ & 70 & Full sun & Low & $\begin{array}{l}\text { Spreading crown with serrated, heart-shaped leaves with a } \\
\text { rough upper surface. Relatively tasteless, copious amounts } \\
\text { of fruit attract birds and other wildlife. }\end{array}$ \\
\hline $\begin{array}{l}\text { Jamaican } \\
\text { dogwood } \\
\text { Piscidia piscipula }\end{array}$ & $30-50$ & Full sun & High & $\begin{array}{l}\text { Fast-growing, attractive, deciduous tree with dark green } \\
\text { leaves and masses of white flowers prior to leafing out in } \\
\text { spring. All parts of the tree are poisonous. }\end{array}$ \\
\hline $\begin{array}{l}\text { West Indian } \\
\text { cherry } \\
\text { Prunus myrtifolia }\end{array}$ & $20-30$ & $\begin{array}{l}\text { Full sun to } \\
\text { partial shade }\end{array}$ & Low & $\begin{array}{l}\text { Accent or specimen tree with shiny, lime green, evergreen } \\
\text { leaves and reddish-gray trunk bearing shallow cracks. Fruit } \\
\text { is food source for many birds and small animals. Leaves } \\
\text { and seeds poisonous to humans. }\end{array}$ \\
\hline $\begin{array}{l}\text { Laurel oak* }^{*} \\
\text { Quercus laurifolia }\end{array}$ & $60-70$ & Full sun & High & $\begin{array}{l}\text { Large, semi-evergreen tree with lance-shaped leaves, } \\
\text { shiny green above and pale green below. Deeply furrowed } \\
\text { bark. Pyramidal when young; rounded when full grown. } \\
\text { Faster growing but weaker and shorter-lived than live oak. } \\
\text { Very sensitive to being planted too deep. }\end{array}$ \\
\hline
\end{tabular}


Table 3. Native medium and large trees for south Florida, listed in alphabetical order by scientific name. (Note: * indicates plants commonly available in native nurseries in south Florida.)

\begin{tabular}{|c|c|c|c|c|}
\hline $\begin{array}{l}\text { Common name } \\
\text { Scientific name }\end{array}$ & $\begin{array}{l}\text { Size } \\
\text { (feet) }\end{array}$ & $\begin{array}{l}\text { Light } \\
\text { preference }\end{array}$ & $\begin{array}{c}\text { Salt } \\
\text { tolerance }\end{array}$ & Comments \\
\hline $\begin{array}{l}\text { Live oak * } \\
\text { Quercus virginiana }\end{array}$ & $40-50$ & Full sun & High & $\begin{array}{l}\text { Impressive, undemanding, tree. Not for small lots. } \\
\text { Caterpillars, insect galls, and root rot sometimes a } \\
\text { problem. Needs early pruning to properly shape into tree. }\end{array}$ \\
\hline $\begin{array}{l}\text { Soapberry } \\
\text { Sapindus } \\
\text { saponaria }\end{array}$ & $20-60$ & Full sun & High & $\begin{array}{l}\text { Broad crown, dense foliage, and attractive yellow fruit. } \\
\text { Excellent shade tree for small yards. Seeds poisonous. }\end{array}$ \\
\hline $\begin{array}{l}\text { Mastic * } \\
\text { Sideroxylon } \\
\text { foetidissimum }\end{array}$ & $50-70$ & Full sun & Moderate & $\begin{array}{l}\text { Large tree with pale, bright yellow, fragrant flowers in } \\
\text { summer, and fruit in late winter. Excellent resistance to } \\
\text { storms. Mastichodendron foetidissimum is a synonym. }\end{array}$ \\
\hline $\begin{array}{l}\text { Willow bustic * } \\
\text { Sideroxylon } \\
\text { salicifolium }\end{array}$ & $10-30$ & $\begin{array}{l}\text { Full sun to } \\
\text { partial shade }\end{array}$ & Low & See description in Table 2 above. \\
\hline $\begin{array}{l}\text { Paradise tree * } \\
\text { Simarouba glauca }\end{array}$ & $30-50$ & Full sun & Low & $\begin{array}{l}\text { Large, elegant tree with a broad crown of dark green, } \\
\text { glossy leaves divided into } 10-14 \text { stiff, rounded leaflets. } \\
\text { Separate male and female trees. Females produce an } \\
\text { abundance of small fruit. Requires excellent drainage. }\end{array}$ \\
\hline $\begin{array}{l}\text { Mahogany * } \\
\text { Swietenia } \\
\text { mahagoni }\end{array}$ & $40-75$ & Full sun & Moderate & $\begin{array}{l}\text { Attractive, storm-resistant tree. Prized for their wood, which } \\
\text { is a deep red color. Falling woody cones can damage } \\
\text { vehicles. Foliage occasionally chewed by insects. }\end{array}$ \\
\hline $\begin{array}{l}\text { Florida trema } \\
\text { Trema micranthum }\end{array}$ & $5-30$ & $\begin{array}{l}\text { Full sun to } \\
\text { partial shade }\end{array}$ & Low & See description in Table 2 above. \\
\hline
\end{tabular}

Table 4. Native palms and our single native cycad for south Florida, listed in alphabetical order by scientific name. (Note: * indicates plants commonly available in native nurseries in south Florida. Note also that all of these palms are resistant to lethal yellowing disease.)

\begin{tabular}{||l|c|c|c|l||}
\hline \hline $\begin{array}{l}\text { Common name } \\
\text { Scientific name }\end{array}$ & $\begin{array}{c}\text { Size } \\
\text { (feet) }\end{array}$ & $\begin{array}{c}\text { Light } \\
\text { preference }\end{array}$ & $\begin{array}{c}\text { Salt } \\
\text { tolerance }\end{array}$ & \multicolumn{1}{||}{ Comments } \\
\hline $\begin{array}{l}\text { Silver palm * } \\
\text { Coccothrinax } \\
\text { argentata }\end{array}$ & $3-8$ & Full sun & High & $\begin{array}{l}\text { Small, slow-growing palm with round, palmate leaves } \\
\text { that are dark, shiny green above and silvery below. } \\
\text { Small, purplish-black fruit produced nearly } \\
\text { year-round. }\end{array}$ \\
\hline $\begin{array}{l}\text { Buccaneer palm, } \\
\text { Sargentcherry } \\
\text { palm * } \\
\text { Pseudophoenix } \\
\text { sargentii }\end{array}$ & 10 & Full sun & High & $\begin{array}{l}\text { Slow-growing feather leaf palm. Grows naturally in } \\
\text { sandy or limestone soils in areas that receive little } \\
\text { rainfall. Gray trunk and prominent gray-green } \\
\text { crownshaft, topped by sparse crown of } \\
\text { silvery-blue-green leaves. Produces grape-sized, red } \\
\text { fruit. Considered endangered in Florida, but common } \\
\text { in the nursery trade. }\end{array}$ \\
\hline \hline
\end{tabular}


Table 4. Native palms and our single native cycad for south Florida, listed in alphabetical order by scientific name. (Note: * indicates plants commonly available in native nurseries in south Florida. Note also that all of these palms are resistant to lethal yellowing disease.)

\begin{tabular}{||l|c|c|c|l||}
\hline \hline $\begin{array}{l}\text { Common name } \\
\text { Scientific name }\end{array}$ & $\begin{array}{c}\text { Size } \\
\text { (feet) }\end{array}$ & $\begin{array}{c}\text { Light } \\
\text { preference }\end{array}$ & $\begin{array}{c}\text { Salt } \\
\text { tolerance }\end{array}$ & \multicolumn{1}{|c||}{ Comments } \\
\hline $\begin{array}{l}\text { Scrub palmetto } \\
\text { Sabal etonia }\end{array}$ & $3-5$ & Full sun & Moderate & $\begin{array}{l}\text { Small, trunkless, solitary, shrub-like fan palm native to } \\
\text { central Florida. Bears large clusters of small, purplish } \\
\text { black fruit. Grows best in sandy soils. }\end{array}$ \\
\hline $\begin{array}{l}\text { Dwarf blue } \\
\text { palmetto * } \\
\text { Sabal minor }\end{array}$ & $2-4$ & Partial shade & Moderate & $\begin{array}{l}\text { Small, trunkless, shrub-like palm native to central and } \\
\text { north Florida. Resembles S. etonia, but smaller and } \\
\text { with bluish-green leaves. Can tolerate wet soils. }\end{array}$ \\
\hline $\begin{array}{l}\text { Cabbage palm * } \\
\text { Sabal palmetto }\end{array}$ & $25-50$ & $\begin{array}{c}\text { Full sun to } \\
\text { partial shade }\end{array}$ & High & $\begin{array}{l}\text { Common tall palm with rounded crown of fan-shaped } \\
\text { leaves and smooth or rough trunk. Highly adaptable. }\end{array}$ \\
\hline $\begin{array}{l}\text { Saw palmetto * } \\
\text { Serenoa repens }\end{array}$ & $3-8$ & $\begin{array}{c}\text { Full sun to } \\
\text { partial shade }\end{array}$ & High & See description in Table 2 above. \\
\hline $\begin{array}{l}\text { Keys thatch palm * } \\
\text { Thrinax morrissii }\end{array}$ & 15 & $\begin{array}{c}\text { Full sun to } \\
\text { partial sun }\end{array}$ & High & $\begin{array}{l}\text { Grows naturally in alkaline soils, sometimes on } \\
\text { limestone outcrops. Fan-shaped leaves are } \\
\text { bluish-green above and silvery below. Small white fruit } \\
\text { produced in the fall. }\end{array}$ \\
\hline $\begin{array}{l}\text { Florida thatch palm } \\
\text { * Thrinax radiata }\end{array}$ & 25 & $\begin{array}{l}\text { Full sun to } \\
\text { partial sun }\end{array}$ & High & $\begin{array}{l}\text { Grows naturally in sand or on limestone. Differs from } T . \\
\text { morrissii by having leaves that are green on both sides. }\end{array}$ \\
\hline $\begin{array}{l}\text { Florida coontie * } \\
\text { Zamia floridana }\end{array}$ & $1-5$ & $\begin{array}{l}\text { Full sun to } \\
\text { shade }\end{array}$ & High & $\begin{array}{l}\text { Florida's only native cycad. See full description in } \\
\text { Table 2 above. }\end{array}$ \\
\hline \hline
\end{tabular}

Table 5. Native ornamental grasses, ferns, and groundcovers for south Florida, listed in alphabetical order by scientific name. (Note: * indicates plants commonly available in native nurseries in south Florida.)

\begin{tabular}{|c|c|c|c|c|}
\hline $\begin{array}{l}\text { Common name } \\
\text { Scientific name }\end{array}$ & Size & $\begin{array}{l}\text { Light } \\
\text { preference }\end{array}$ & $\begin{array}{c}\text { Salt } \\
\text { tolerance }\end{array}$ & Comments \\
\hline $\begin{array}{l}\text { Quail berry, ground } \\
\text { holly * } \\
\text { Crossopetalum } \\
\text { ilicifolium }\end{array}$ & $\begin{array}{c}12 \\
\text { inches }\end{array}$ & $\begin{array}{l}\text { Full sun to } \\
\text { partial shade }\end{array}$ & Low & $\begin{array}{l}\text { Prostrate, evergreen shrubby groundcover with small, } \\
\text { holly-like, spiny leaves and attractive red berries. }\end{array}$ \\
\hline $\begin{array}{l}\text { Penny grass } \\
\text { Dichondra } \\
\text { carolinensis }\end{array}$ & $\begin{array}{c}2 \\
\text { inches }\end{array}$ & Full sun & High & $\begin{array}{l}\text { Low-growing groundcover for edges and mass plantings. } \\
\text { Inconspicuous flowers and evergreen foliage. Prefers moist } \\
\text { soil. }\end{array}$ \\
\hline $\begin{array}{l}\text { Elliottlove grass } \\
\text { Eragrostis elliottii }\end{array}$ & $\begin{array}{l}12-24 \\
\text { inches }\end{array}$ & $\begin{array}{l}\text { Full sun to } \\
\text { light shade }\end{array}$ & Low & $\begin{array}{l}\text { Small, fine-textured bunchgrass with beautiful, silvery-blue } \\
\text { leaves and masses of tiny, delicate flower spikes. }\end{array}$ \\
\hline $\begin{array}{l}\text { Purple love grass * } \\
\text { Eragrostis } \\
\text { spectabilis }\end{array}$ & $\begin{array}{l}12-18 \\
\text { inches }\end{array}$ & Full sun & Low & $\begin{array}{l}\text { Clumping ornamental grass with soft, light green, } \\
\text { fine-textured leaves and delicate panicles of tiny, } \\
\text { reddish-purple flowers held high above foliage. Grows well } \\
\text { in hot, dry sites; will not tolerate wet, shady sites. }\end{array}$ \\
\hline
\end{tabular}


Table 5. Native ornamental grasses, ferns, and groundcovers for south Florida, listed in alphabetical order by scientific name. (Note: * indicates plants commonly available in native nurseries in south Florida.)

\begin{tabular}{|c|c|c|c|c|}
\hline $\begin{array}{l}\text { Common name } \\
\text { Scientific name }\end{array}$ & Size & $\begin{array}{l}\text { Light } \\
\text { preference }\end{array}$ & $\begin{array}{l}\text { Salt } \\
\text { tolerance }\end{array}$ & Comments \\
\hline $\begin{array}{l}\text { Sunshine mimosa } \\
\text { Mimosa strigillosa }\end{array}$ & $\begin{array}{c}6-9 \\
\text { inches }\end{array}$ & Full sun & Moderate & $\begin{array}{l}\text { Prostrate, carpeting perennial with creeping, widely } \\
\text { spreading stems. Leaves are finely divided and bipinnate. } \\
\text { Flowers are pink powderpuffs. Can become weedy. }\end{array}$ \\
\hline $\begin{array}{l}\text { Long-stalked } \\
\text { stopper } \\
\text { Mosiera longipes }\end{array}$ & $\begin{array}{c}1.5-3 \\
\text { feet }\end{array}$ & $\begin{array}{l}\text { Full sun to } \\
\text { light shade }\end{array}$ & Low & See description in Table 2 above. \\
\hline $\begin{array}{l}\text { Muhly grass * } \\
\text { Muhlenbergia } \\
\text { capillaries }\end{array}$ & $2-3$ feet & $\begin{array}{l}\text { Full sun to } \\
\text { partial shade }\end{array}$ & High & $\begin{array}{l}\text { Elegant, compact, tuft-forming ornamental grass with fine, } \\
\text { feather-like, pink flower spikes held high above the leaves. }\end{array}$ \\
\hline $\begin{array}{l}\text { Native sword fern } \\
\text { Nephrolepis } \\
\text { biserrata }\end{array}$ & $3-4$ feet & $\begin{array}{l}\text { Partial to full } \\
\text { shade }\end{array}$ & Low & $\begin{array}{l}\text { Tall fern with elegant leaves. Makes an excellent } \\
\text { groundcover or informal hedge. Commonly available form is } \\
\text { called fishtail fern }\end{array}$ \\
\hline $\begin{array}{l}\text { Baby rubber plant * } \\
\text { Peperomia } \\
\text { obtusifolia }\end{array}$ & $\begin{array}{l}12-18 \\
\text { inches }\end{array}$ & $\begin{array}{l}\text { Partial to full } \\
\text { shade }\end{array}$ & Low & $\begin{array}{l}\text { Bushy groundcover with fleshy leaves and occasional } \\
\text { spikes of minute flowers. Plain green or variegated cultivars, } \\
\text { the latter with leaves marbled in gray-green and cream or } \\
\text { gold. }\end{array}$ \\
\hline $\begin{array}{l}\text { Silkgrass * } \\
\text { Pityopsis } \\
\text { graminifolia }\end{array}$ & $2-3$ feet & Full sun & Low & See description in Table 1 above. \\
\hline $\begin{array}{l}\text { Pineland brake } \\
\text { Pteris bahamensis }\end{array}$ & $1-2$ feet & $\begin{array}{l}\text { Full sun to } \\
\text { light shade }\end{array}$ & Low & $\begin{array}{l}\text { Elegant fern that grows in tidy clumps. Old, brown leaves } \\
\text { accumulate after a while. Entire plant can be cut back to the } \\
\text { ground in early spring to rejuvenate the plant. }\end{array}$ \\
\hline $\begin{array}{l}\text { Sea purslane } \\
\text { Sesuvium } \\
\text { portulacastrum }\end{array}$ & $\begin{array}{c}6-8 \\
\text { inches }\end{array}$ & Full sun & High & $\begin{array}{l}\text { Sprawling, succulent groundcover with small, light green to } \\
\text { yellow, bead-like leaves and tiny purplish-pink flowers. Good } \\
\text { for beach-front properties. }\end{array}$ \\
\hline $\begin{array}{l}\text { Lopsided Indian } \\
\text { grass } \\
\text { Sorghastrum } \\
\text { secundum }\end{array}$ & $\begin{array}{l}24-48 \\
\text { inches }\end{array}$ & $\begin{array}{l}\text { Full sun to } \\
\text { light shade }\end{array}$ & Low & $\begin{array}{l}\text { Beautiful, clump-forming grass with fairly nondescript leaves } \\
\text { in spring and summer. In late summer or early fall, however, } \\
\text { 4- to 6-foot spikes emerge containing multicolored flowers } \\
\text { along only one side of the spike. }\end{array}$ \\
\hline $\begin{array}{l}\text { Sand cordgrass * } \\
\text { Spartina bakeri }\end{array}$ & $4-6$ feet & Full sun & High & $\begin{array}{l}\text { Large, bunch-forming grass with thin, brownish-green, } \\
\text { rolled, wire-like, sand-papery leaves. Highly adaptable and } \\
\text { underutilized. Good for beach-front. }\end{array}$ \\
\hline $\begin{array}{l}\text { Pineland dropseed } \\
\text { Sporobolus junceus }\end{array}$ & $1-3$ feet & Full sun & Moderate & $\begin{array}{l}\text { One of the most beautiful and ornamental of all grasses. } \\
\text { Forms large, hemispherical clumps of tiny, wire-like leaves } \\
\text { of blue-green or silvery green. Flower spikes are tall and } \\
\text { bear numerous tiny, reddish-colored flowers. }\end{array}$ \\
\hline $\begin{array}{l}\text { Blue porterweed* } \\
\text { Stachytarpheta } \\
\text { jamaicensis }\end{array}$ & $\begin{array}{l}12-36 \\
\text { inches }\end{array}$ & $\begin{array}{l}\text { Full sun to } \\
\text { partial shade }\end{array}$ & Moderate & See description in Table 1 above. \\
\hline $\begin{array}{l}\text { Fakahatchee grass } \\
\text { * Tripsacum } \\
\text { dactyloides }\end{array}$ & $4-5$ feet & $\begin{array}{l}\text { Full sun to } \\
\text { partial shade }\end{array}$ & Moderate & $\begin{array}{l}\text { Large grass with rich green foliage that erupts from } \\
\text { fountain-like clumps. Distinctive flowers rise above leaves } \\
\text { on slender stems in midsummer. Easy to grow. Virtually free } \\
\text { of pests. }\end{array}$ \\
\hline
\end{tabular}


Table 5. Native ornamental grasses, ferns, and groundcovers for south Florida, listed in alphabetical order by scientific name. (Note: * indicates plants commonly available in native nurseries in south Florida.)

\begin{tabular}{||l|c|c|c|l||}
\hline \hline $\begin{array}{c}\text { Common name } \\
\text { Scientific name }\end{array}$ & Size & $\begin{array}{c}\text { Light } \\
\text { preference }\end{array}$ & $\begin{array}{c}\text { Salt } \\
\text { tolerance }\end{array}$ & \multicolumn{1}{c||}{ Comments } \\
\hline $\begin{array}{l}\text { Florida gamma } \\
\text { grass * } \\
\text { Tripsacum floridana }\end{array}$ & $2-4$ feet & $\begin{array}{c}\text { Full sun to } \\
\text { partial shade }\end{array}$ & Moderate & $\begin{array}{l}\text { Similar to T. dactyloides, but smaller and with finer-textured } \\
\text { leaves. Also called dwarf Fakahatchee grass. }\end{array}$ \\
\hline $\begin{array}{l}\text { Florida coontie * } \\
\text { Zamia floridana }\end{array}$ & $1-5$ feet & $\begin{array}{c}\text { Full sun to } \\
\text { shade }\end{array}$ & High & See description in Table 2 above. \\
\hline \hline
\end{tabular}

Table 6. Native vines for south Florida, listed in alphabetical order by scientific name. (Note: * indicates plants commonly available in native nurseries in south Florida.)

\begin{tabular}{|c|c|c|c|c|}
\hline $\begin{array}{l}\text { Common name } \\
\text { Scientific name }\end{array}$ & Size & $\begin{array}{l}\text { Light } \\
\text { preference }\end{array}$ & $\begin{array}{l}\text { Salt } \\
\text { tolerance }\end{array}$ & Comments \\
\hline $\begin{array}{l}\text { Beach bean } \\
\text { Canavalia rosea }\end{array}$ & $\begin{array}{c}6-12 \\
\text { inches }\end{array}$ & Full sun & High & $\begin{array}{l}\text { Attractive purple flowers. Excellent groundcover for } \\
\text { beach-front locations, although it can grow into } \\
\text { surrounding shrubs or over fences. }\end{array}$ \\
\hline $\begin{array}{l}\text { Limber caper * } \\
\text { Capparis flexuosa }\end{array}$ & $\begin{array}{c}12-25 \\
\text { feet }\end{array}$ & $\begin{array}{l}\text { Full sun to } \\
\text { full shade }\end{array}$ & High & See description in Table 2 above. \\
\hline $\begin{array}{l}\text { Butterfly pea } \\
\text { Centrosema } \\
\text { virginianum }\end{array}$ & $3-12$ feet & Full sun & Low & $\begin{array}{l}\text { Easily cultivated vine with delicate, finely divided leaves } \\
\text { bearing three long, narrow lobes. Flowers are } \\
\text { purplish-pink and pea-like in appearance. }\end{array}$ \\
\hline $\begin{array}{l}\text { Rubber vine, } \\
\text { devilpotato } \\
\text { Echites umbellata }\end{array}$ & $\begin{array}{c}10-30 \\
\text { feet }\end{array}$ & Full sun & Low & $\begin{array}{l}\text { Evergreen, twining, climbing vine with dark green, glossy } \\
\text { leaves and beautiful tubular white flowers. All parts of this } \\
\text { plant are highly toxic. }\end{array}$ \\
\hline $\begin{array}{l}\text { Railroad vine } \\
\text { Ipomoea } \\
\text { pes-caprae }\end{array}$ & $\begin{array}{c}3-8 \\
\text { inches }\end{array}$ & Full sun & High & $\begin{array}{l}\text { Course, sprawling vine with smooth, fleshy leaves and } \\
\text { large, rosy pink, flowers. }\end{array}$ \\
\hline $\begin{array}{l}\text { Beach morning } \\
\text { glory } \\
\text { Ipomoea } \\
\text { stolonifera }\end{array}$ & 6 inches & Full sun & High & $\begin{array}{l}\text { Twining, viney groundcover grows over sand dunes. } \\
\text { Blooms from spring to fall, with white flowers opening each } \\
\text { morning and closing in the afternoon. Ideal for beach-front. }\end{array}$ \\
\hline $\begin{array}{l}\text { Key morning glory } \\
\text { Jacquemontia } \\
\text { pentanthos }\end{array}$ & $\begin{array}{c}10-20 \\
\text { feet }\end{array}$ & Full sun & High & $\begin{array}{l}\text { Twining, rambling vine with type flowers in spring, summer, } \\
\text { and fall. Native to Florida Keys, so is susceptible to frost. } \\
\text { Also called skyblue clustervine. }\end{array}$ \\
\hline $\begin{array}{l}\text { Coral honeysuckle } \\
\text { * } \\
\text { Lonicera } \\
\text { sempervirens }\end{array}$ & $\begin{array}{c}10-15 \\
\text { feet }\end{array}$ & $\begin{array}{l}\text { Full sun to } \\
\text { partial shade }\end{array}$ & Low & $\begin{array}{l}\text { Showy evergreen vine that blooms heavily in the spring } \\
\text { with clusters of tubular, orange-red flowers that attract } \\
\text { butterflies and hummingbirds. }\end{array}$ \\
\hline $\begin{array}{l}\text { Morinda, redgal } \\
\text { Morinda royoc }\end{array}$ & 5-10 feet & $\begin{array}{l}\text { Full sun to } \\
\text { partial shade }\end{array}$ & High & See description in Table 2 above. \\
\hline
\end{tabular}


Table 6. Native vines for south Florida, listed in alphabetical order by scientific name. (Note: * indicates plants commonly available in native nurseries in south Florida.)

\begin{tabular}{||l|c|c|c|l||}
\hline \hline $\begin{array}{l}\text { Common name } \\
\text { Scientific name }\end{array}$ & Size & $\begin{array}{c}\text { Light } \\
\text { preference }\end{array}$ & $\begin{array}{c}\text { Salt } \\
\text { tolerance }\end{array}$ & \multicolumn{1}{|c||}{ Comments } \\
\hline $\begin{array}{l}\text { Passion flower * } \\
\text { Passiflora } \\
\text { incarnata }\end{array}$ & 5 -10 feet & $\begin{array}{c}\text { Full sun to } \\
\text { partial shade }\end{array}$ & Low & $\begin{array}{l}\text { Vine with shiny green leaves and large, showy, fringed } \\
\text { white/purple flowers followed by medium-sized, purple } \\
\text { fruit. Highly attractive to butterflies as a larval food plant. }\end{array}$ \\
\hline $\begin{array}{l}\text { Corky-stemmed } \\
\text { passion flower } \\
\text { Passiflora } \\
\text { suberosa }\end{array}$ & 20 feet & $\begin{array}{c}\text { Full sun to } \\
\text { partial shade }\end{array}$ & Low & $\begin{array}{l}\text { Vine with shiny green leaves and very small, greenish } \\
\text { yellow flowers followed by small, purple fruit. Highly } \\
\text { attractive to tropical zebra butterflies as a larval food plant. }\end{array}$ \\
\hline $\begin{array}{l}\text { Wild allamanda * } \\
\text { Pentalinon luteum }\end{array}$ & 50 feet & $\begin{array}{c}\text { Full sun to } \\
\text { partial shade }\end{array}$ & Moderate & $\begin{array}{l}\text { Fast growing, twining vine to 50 inches in length. Lustrous, } \\
\text { elliptical leaves with lighter colored midribs. Blooms } \\
\text { year-round. Blossoms bright yellow, bell-shaped. } \\
\text { Urechites lutea is a synonym. }\end{array}$ \\
\hline $\begin{array}{l}\text { Rubber or } \\
\text { mangrove vine } \\
\begin{array}{l}\text { Rhabdadenia } \\
\text { biflora }\end{array}\end{array}$ & 12 feet & $\begin{array}{c}\text { Full sun to } \\
\text { partial shade }\end{array}$ & High & $\begin{array}{l}\text { Robust, twining vine with dark green leaves and beautiful, } \\
\text { paper-white flowers with yellow centers and edges tinged } \\
\text { with pink. Often found in association with mangroves, but it } \\
\text { does not require such conditions to grow well. }\end{array}$ \\
\hline $\begin{array}{l}\text { Mistletoe cactus } \\
\text { Rhipsalis baccifera }\end{array}$ & 6 feet & Light to full \\
shade & High & $\begin{array}{l}\text { Hanging epiphytic cactus with pencil-thin, yellowish stems } \\
\text { 2 feet or more in length. Bears small, white flowers and } \\
\text { small, white berries with black seeds. }\end{array}$ \\
\hline
\end{tabular}

